\title{
Quantum Bounds on Bell inequalities
}

\author{
Károly F. Pál* and Tamás Vértes \\ Institute of Nuclear Research of the Hungarian Academy of Sciences \\ H-4001 Debrecen, P.O. Box 51, Hungary
}

(Dated: September 25, 2018)

\begin{abstract}
We have determined the maximum quantum violation of 241 tight bipartite Bell inequalities with up to five two-outcome measurement settings per party by constructing the appropriate measurement operators in up to six-dimensional complex and eight-dimensional real component Hilbert spaces using numerical optimization. Out of these inequalities 129 has been introduced here. In 43 cases higher dimensional component spaces gave larger violation than qubits, and in 3 occasions the maximum was achieved with six-dimensional spaces. We have also calculated upper bounds on these Bell inequalities using a method proposed recently. For all but 20 inequalities the best solution found matched the upper bound. Surprisingly, the simplest inequality of the set examined, with only three measurement settings per party, was not among them, despite the high dimensionality of the Hilbert space considered. We also computed detection threshold efficiencies for the maximally entangled qubit pair. These could be lowered in several instances if degenerate measurements were also allowed.

PACS numbers: 03.65.Ud, 03.67.-a
\end{abstract}

\section{INTRODUCTION}

Suppose two classical systems which are separated from each other. Let us make some local measurements on them. Then the results of these measurements may be correlated, which may be explained by shared randomness experienced in the past. For a given number of possible measurement choices (inputs) and results (outputs) the set of correlations forms a polytope whose finite number of vertices correspond to all the deterministic assignments of outputs to inputs. The facets of the polytope, which form the boundary of the classical region, correspond to tight Bell inequalities [1, 2]. Thus Bell inequalities has no a priori relation to quantum physics. However, quantum physics violates them, which has been verified experimentally in numerous occasions up to some technical loopholes (see e.g., [3]). Indeed, this makes Bell inequalities very interesting.

On the other hand, one may ask what is the achievable set of correlations if one allows the two parties to share quantum resources as well over shared randomness. This is a convex set, such as for the classical case, however it cannot be described by a finite number of extreme points [4]. Nevertheless, one can construct so called quantumBell inequalities which bound the correlations achievable by quantum physics [4 6] (see also item 5 in Sec. III.C of Ref. [7]). A simple way to form quantum-Bell inequalities is to use the coefficients of known Bell inequalities, and determine the maximum value one can get by performing measurements on quantum systems of arbitrary dimensions.

The simplest result in this respect is the Tsirelson bound [8] stating that quantum correlations cannot vio-

\footnotetext{
*Electronic address: kfpal@atomki.hu

${ }^{\dagger}$ Electronic address: tvertesi@dtp.atomki.hu
}

late the CHSH inequality [2] by more than $(\sqrt{2}-1) / 2$. Note, however that the requirement of non-signaling alone allows a higher bound of 1 [9, 10] (the maximum allowed value in a local classical theory is 0 ). In the present paper we stick to the bipartite two-outcome scenario and we consider inequalities with more than two measurement settings having nontrivial local marginals as well.

In particular, in this paper we test 241 bipartite tight Bell inequalities with two-valued observables with up to five settings. These Bell inequalities have been collected from various sources: from the list of Ref. [11] containing 89 inequalities labelled by $A_{i}, i=3, \ldots, 89$, from the list of Ref. [12] comprising 31 inequalities with up to four settings per party. 18 of them, labelled by $I_{4422}^{i}, i=$ $3, \ldots, 20$ are introduced by that paper. The simplest nontrivial inequality $I_{3322}$ beside the CHSH inequality was presented first by Froissart 13 in 1981 and more recently it was reintroduced by Sliwa 14] and by Collins and Gisin [15]. The rest of them, namely, $I_{3422}^{1,2,3}$ and $I_{4422}^{1}$ were presented in Ref. [15], $I_{4422}^{2}$ in [16], $A_{5,6}$ and $A I I_{1,2}$ in [17], while $A S_{1,2}$ in [7]. These lists were also considered by us previously [18]. Finally, a list of 129 tight bipartite Bell inequalities with four settings per party is constructed in the present paper (labelled by $J_{4422}^{i}, i=1, \ldots, 129$ ). We test numerically these Bell inequalities and give the bound on them achievable by quantum systems, thereby generating quantum-Bell inequalities. On one hand, we used numerical optimization in higher dimensional (up to six complex and eight real) component Hilbert spaces to calculate a lower bound on maximal Bell violation. On the other hand, the hierarchy of tests of Refs. [19, 20] allows us to give upper bounds on the violation achievable by quantum systems. We found in a surprisingly many cases that the lower and upper bounds coincide indicating that the quantum bound we have found is tight. Numerics suggests that in some cases even five- 
dimensional local Hilbert spaces (i.e., 25-dimensional full Hilbert space) were not enough to achieve the maximum quantum value. This result can be interpreted through the recent concept of dimension witness 21], which is a certain kind of generalization of Bell inequalities which enables to measure the dimension of the Hilbert space (see also Refs. [22, 23] for recent works using this concept). In particular, a $d$-dimensional Hilbert space witness allows to distinguish the strength of correlations of measurement outcomes on members of composite systems that can be obtained in $d$-dimensional component Hilbert spaces from the ones which are achievable only in higher dimensions. Our result thus indicates a fivedimensional witness, which improves on our recent result [18], where we found three-dimensional witness. We also made a study, given these tight Bell inequalities, from a different perspective. Namely, we computed the threshold detection efficiency for a maximally entangled qubit pair required to close the detection loophole in the symmetric (Alice and Bob having the same detection efficiency) and in the asymmetric cases (Alice has perfect detection efficiency) by considering degenerate measurements (having deterministic outcomes) as well. Surprisingly, in almost all cases degenerate measurements were required to achieve the lowest threshold efficiency in both the symmetric and asymmetric situations. However, let us note, that these cases with degenerate measurements could be traced back to ones with non-degenerate measurements, which achieve the same threshold value. In the asymmetric case we could improve on the best known results from the literature [12, 24], by carrying out only non-degenerate measurements.

The rest of the paper is organized as follows. In Sec. II we describe methods to find tight two-outcome bipartite Bell inequalities, and we show the 129 inequivalent examples we have got this way. For all of them the classical limit is zero, and we have given a symmetric form, whenever there exists one. In Sec. III it is explained how we constructed the maximum quantum violating solution in real and complex six-, and real eight-dimensional component Hilbert spaces. In Sec. IV we present briefly the method of Navascués et al. [19, 20] to obtain upper limit for the maximum quantum violation of twoparty two-outcome Bell inequalities. In Sec. V we give the maximum quantum value we have derived, the size of the Hilbert spaces required, and the dimensionalities of the projectors used as the measurement operators for all the 241 Bell inequalities considered. We also show when this value reaches the upper bound. For those cases the value given is the absolute quantum bound. We discuss the results obtained. In Section VI the minimal detection efficiencies are computed for a maximally entangled pair of qubits considering degenerate measurements as well. More details of the results including the matrices of the optimal operators and the Schmidt coefficients of the states are presented in the web site [52]. SectionVII summarizes the results of the paper.

\section{METHODS FOR GENERATING TIGHT BELL INEQUALITIES}

We consider a typical experiment to test for correlations. Imagine a system, either quantum or classical, which is composed of two subsystems, $A$ and $B$. Suppose that Alice has $m_{A}$ choices of two-valued measurements on $A$, while Bob has $m_{B}$ choices of two-valued measurements on $B$. Let us refer to this situation with the notation $m_{A} m_{B} 22$. The outcome of the measurements is one of two values labelled by the symbols 1 and 0 . The experiment is repeated many times with different choices of measurement settings so as to get accurate probabilities. The result of such a correlation experiment can be thought of as a vector $\vec{p}$ in a $d$ dimensional space, where $d=m_{A}+m_{B}+m_{A} m_{B}$. The components $p_{A_{i}}, p_{B_{j}}$ and $p_{A_{i} B_{j}}$ (for $1 \leq i \leq m_{A}$ and $1 \leq j \leq m_{B}$ ) of $\vec{p}$ represent the probability that Alice gets 1 for measurement setting $i$, Bob gets 1 for measurement setting $j$, and that both Alice and Bob get 1 for setting $i$ and $j$ on the respective sides.

In a quantum correlation experiment Alice and Bob share a quantum state $\rho$, and the probabilities can be expressed by the formulae

$$
\begin{aligned}
p_{A_{i}} & =\operatorname{Tr}\left[\rho\left(A_{i} \otimes I_{B}\right)\right], \\
p_{B_{j}} & =\operatorname{Tr}\left[\rho\left(I_{B} \otimes B_{j}\right)\right], \\
p_{A_{i} B_{j}} & =\operatorname{Tr}\left[\rho\left(A_{i} \otimes B_{j}\right)\right],
\end{aligned}
$$

where $I_{A}\left(I_{B}\right)$ denotes the unity operator acting on the Hilbert space of Alice (Bob), while $A_{i}$ and $B_{j}$ are the $\{1,0\}$ valued observables (i.e., projectors) measured by Alice and Bob, respectively. Note, that for our case of binary outcomes it has been shown [25] that projective measurements can reproduce the set of all quantum correlations.

On the other hand, the result of a classical correlation experiment must correspond to a probability distribution represented as a convex sum over all deterministic configurations. A deterministic configuration assigns the outcomes $\{1,0\}$ to each of the $m_{A}+m_{B}$ measurements, in which case the joint probabilities factor into two local probability distributions, $p_{A_{i} B_{j}}=p_{A_{i}} p_{B_{j}}$. Thus, the set of vectors $\vec{p}$ which are possible results of a correlation experiment forms a $d=\left(m_{A}+m_{B}+m_{A} m_{B}\right)$-dimensional polytope $P$ [26]. Froissart [13] has shown that in the general $m_{A} m_{B} 22$ case the set of all $n=2^{m_{A}+m_{B}}$ points $\vec{p}_{v}, v=1, \ldots, n$ corresponding to deterministic configurations are actually the extremal vertices of this $d$ dimensional polytope $P$. The experimental result has a locally causal model if and only if the corresponding point is located inside the polytope $P$. A Bell inequality is a linear inequality satisfied by all the points in such a polytope. A tight Bell inequality, which is the most useful to detect non-local correlations, defines a facet of this polytope. In our particular formulation we represent 
a Bell inequality in the form

$$
\sum_{i=1}^{m_{A}} b_{A_{i}} p_{A_{i}}+\sum_{j=1}^{m_{B}} b_{B_{j}} p_{B_{j}}+\sum_{i=1}^{m_{A}} \sum_{j=1}^{m_{B}} b_{A_{i} B_{j}} p_{A_{i} B_{j}} \leq b_{0},
$$

where the sums run over all measurements of Alice and Bob, and the coefficients $b$ 's are suitably chosen real numbers. The simplest nontrivial tight Bell inequality (with the smallest values of $m_{A}=2$ and $m_{B}=2$ ) is the CHSH inequality [2]

$$
-p_{A_{1}}-p_{B_{1}}+p_{A_{1} B_{1}}+p_{A_{1} B_{2}}+p_{A_{2} B_{1}}-p_{A_{2} B_{2}} \leq 0 .
$$

Also, note that for a given Bell inequality $\vec{b} \vec{p} \leq b_{0}$, with $\vec{p} \in \mathbb{R}^{d}$ and for a $d$-dimensional polytope $P$, the face represented by the inequality is a facet of that polytope $P$ if and only if the rank of the matrix containing the vertices $p_{v}$ of polytope $P$ which saturate the inequality is $d-1$. This fact enables us to test tightness of Bell inequalities.

Given the vertices $p_{v}$ of polytope $P$ in case $m_{A} m_{B} 22$, our task is to find some facets, which defines tight Bell inequalities. A deterministic algorithm (see for instance the package cdd [27]), which explores systematically all the facets, runs too slowly already for the 4422 case to complete this task. This fact was also mentioned by Collins and Gisin in Ref. [15]. In fact, it has been shown by Pitowsky that this problem for the general $m_{A} m_{B} 22$ case is NP-complete [29]. Therefore, we turn to heuristic algorithms and provide two methods which enable us to produce tight two-outcome bipartite Bell inequalities with $m_{A}=m_{B}=4$ measurement settings. The first one uses some portions from Seidel's shelling algorithm [30], while the second one is based on a reduction of the polytope $P$ to smaller ones. Actually, the second method proves to be useful for a larger number of measurement settings as well. For instance, we found that it also works for the 5522 case.

\section{A. Algorithm: shelling of a Bell polytope}

In this subsection we suitably modify a part of Seidel's shelling algorithm [30]. Consider a $d$-dimensional polytope $P$ corresponding to the case $m_{A} m_{B} 22$ (i.e., a Bell polytope), and let $S$ denote the set of vectors $\vec{p}_{v}$ corresponding to deterministic configurations as defined earlier. Imagine travelling along a directed straight line $L$ that intersects the interior of $P$. Start at a point on $L$ that is in the interior of $P$ and move along $L$. Continue moving until you pass through some facet $F$ and then leave the interior of $P$. This facet $F$ is the first in the shelling order. Continue moving away from $P$ along $L$ and more and more facets will become visible. The order in which these facets appear is the shelling order. This algorithm is deterministic in essence, which makes a shelling of the polytope by travelling along a line and determine the facets of the convex hull as they become visible. However, this algorithm runs too long to complete the task in a reasonable time for polytope $P$ of the case 4422. Therefore, a heuristic algorithm is given which uses a part of Seidel's algorithm:

Suppose we know a priori a single facet $F$ of the polytope $P$ defined by all vertices $\vec{p}_{v}^{F} \in S_{F}$ which lie on the supporting hyperplane of this facet $F$. Define $\vec{a}$ by adding up these vectors $\vec{p}_{v}^{F}$. This sets the direction of the shelling line $L$, parameterized by $x(t)=-\vec{a} / t,-\infty<t<+\infty$. Linear programming can be used to determine for every $\vec{p}_{v} \in S$ the first facet in the shelling that contains $\vec{p}_{v}$. Thus for each $\vec{p}_{v} \in S$ one obtains a tight Bell inequality defined by a facet of $P$. Let us collect these facets, which serve as new inputs to the algorithm. This routine is iterated as long as no more new Bell inequalities are found. However, several of them may turn out to be equivalent, i.e., symmetric under party exchange, observable exchange or relabelling of outcomes (see e.g., 31]). Thus one has to take care of selecting the inequivalent Bell inequalities.

\section{B. Algorithm: slicing a Bell polytope}

Similarly as in $\Pi \mathrm{A}$ we start from an a priori known facet $F$ of a $d$-dimensional polytope $P$ corresponding to a tight Bell inequality, $\vec{b} \vec{p} \leq b_{0}$, where $p \in R^{d}$. In this case for all vertices $\vec{p}_{v} \in S$ corresponding to deterministic configurations the inequality holds by definition. Now let us decrease the value of $b_{0}$ to a smaller value $b_{0}^{*}$. Thereby we obtain two groups of vertices, one $\left(S^{\prime}\right)$ for which $\vec{b} \vec{p} \geq$ $b_{0}^{*}$ holds and one $\left(S^{\prime \prime}\right)$ for which it does not hold.

The convex hull of these vertices form polytopes, designated by $P^{\prime}$ and $P^{\prime \prime}$, respectively. By setting an appropriate value for $b_{0}^{*}$ we may get a polytope $P^{\prime}$ in the same dimension $d$ as for the polytope $P$. Now we take as an input these vertices to the cdd program [27] which generate all the facets of this reduced polytope $P^{\prime}$ (assuming that the number of vertices in $P^{\prime}$ is small enough to be handled by the program). However, not all of the generated facets will be the facets of the original polytope $P$ as well. Thus one must sort out the valid faces which fulfill $\vec{b}^{\prime} \vec{p}_{v} \leq b_{0}^{\prime}$ for all $\vec{p}_{v} \in S$, with $\vec{b}^{\prime}$ and $b_{0}^{\prime}$ defining a facet of $P^{\prime}$. Such as in the algorithm $\amalg A$ these facets, which define tight Bell inequalities, form the input to the new cycle of computations. The computation halts only when no more new Bell inequalities can be produced this way.

\section{Comments specific to the 4422 Bell inequalities}

A crucial point of both algorithms is the right choice of the initial facet. A facet in the $d$-dimensional polytope $P$ is called degenerate if it is created by more than $d$ points. The problem lies in the fact that a Bell polytope $P$ in general, and also in the particular case of 4422 is highly degenerate. However, it is not favorable to start 
TABLE I: Tight bipartite Bell inequalities with four two-outcome measurement settings per party, generated with the methods of Section [I] The coefficients, positive or negative single-digit integers, are shown in the order of $b_{A_{1}}, b_{A_{2}}, b_{A_{3}}, b_{A_{4}}, b_{B_{1}}, b_{B_{2}}$, $b_{B_{3}}, b_{B_{4}}, b_{A_{1} B_{1}}, b_{A_{1} B_{2}}, b_{A_{1} B_{3}}, b_{A_{1} B_{4}}, b_{A_{2} B_{1}}, b_{A_{2} B_{2}}, b_{A_{2} B_{3}}, b_{A_{2} B_{4}}, b_{A_{3} B_{1}}, b_{A_{3} B_{2}}, b_{A_{3} B_{3}}, b_{A_{3} B_{4}}, b_{A_{4} B_{1}}, b_{A_{4} B_{2}}, b_{A_{4} B_{3}}, b_{A_{4} B_{4}}$ (see Eq. (2) ). For each inequality only one of the equivalent forms are shown. The form chosen has classical value zero, and whenever possible, it is symmetric.

$J_{4422}^{1}$ 0-200-2-2-201-11-12121-111011-10

$J_{4422}^{2} \quad 00-1-3-2-20-3-210221-211-2112221$

$J_{4422}^{3}$-2-30-5-10-1-3-321331-321-2113221

$J_{4422}^{4}$-1-20-4-10-1-3-211221-321-2112221

$J_{4422}^{5}-3-40-3-10-1-2-231222-221-3122210$

$J_{4422}^{6}$-2-2-200-2001101-1111121-1-12-10

$J_{4422}^{7} \quad-2-30-4-10-1-1-221122-211-2112220$

$J_{4422}^{8} \quad 0-2000-4-2-2-12-112222-121-2-2-111$

$J_{4422}^{9}-10-1-10-2-3-21101-2122112-21-111$

$J_{4422}^{10}-20-1-20-6-4-323-12-3333132-32-221$

$J_{4422}^{11}$ 0-2000-4-2-3-12-122222-121-2-1-111

$J_{4422}^{12}-30-3-3-30-3-3231-23-33313-22-2321$

$J_{4422}^{13}-3-300-3-30023123-33-213-1-22-2-20$

$J_{4422}^{14}$-1-10-4-10-1-2-211111-211-2112221

$J_{4422}^{15}-2-20-5-10-1-2-321221-211-2113221$

$J_{4422}^{16}-4-200-3-2002213202-212-1-12-2-10$

$J_{4422}^{17}$ 0-4-1-10-4-1-1-2211202212-11121-3

$J_{4422}^{18} \quad-3-3-30-3-3-3013-223033-2321231-5$

$J_{4422}^{19}-1-4-10-1-4-1012-112022-1211121-4$

$J_{4422}^{20}$-4-200-2-1002113202-211-1-11-1-10

$J_{4422}^{21}$-2-30-4-10-1-3-221331-321-2112220

$J_{4422}^{22}$-4-200-4-2002213212-212-1-13-2-10

$J_{4422}^{23}-400-100-1-22212-10-110-211-2111$

$J_{4422}^{24}$ 0-1-2-2-4-20032-1-210-1112113-211

$J_{4422}^{25}-300-100-1-2211100-110-111-2111$

$J_{4422}^{26}-3-100-3-1003112101-111-1-12-1-1-1$

$J_{4422}^{27}$-1-2-30-1-2-30211-21-13213-12-222-2

$J_{4422}^{28}-4-200-4-2003213202-212-1-13-2-1-1$

$J_{4422}^{29}-5-300-5-30043143-13-213-1-24-2-2-2$

$J_{4422}^{30}-2-30-3-10-2-1-121122-211-221122-1$

$J_{4422}^{31}-10-1-30-4-2-112-11-2221121-22-121$

$J_{4422}^{32}-1-2-10-1-2-100011032-212-111-21-1$

$J_{4422}^{33}-3-10-200-1-2121101-110-312-1211$

$J_{4422}^{34}-2-20-3-20-1-2-121111-221-212321-1$

$J_{4422}^{35} \quad 00-1-30-3-1-3-221101-111-3122311$

$J_{4422}^{36}$-3-20-200-1-3121102-220-313-1211

$J_{4422}^{37}-2-10-1-30-2-1021120-211-221211-1$

$J_{4422}^{38}$ 00-3-4-10-3-61-2-12-403412-234132

$J_{4422}^{39} 0-1-3-3-10-2-51-2-12-303412-233121$

$J_{4422}^{40}-2-4-300-4-3-2231-3313313-22-5321$

$J_{4422}^{41}-1-4-10-1-4-10-32112122122-212-20$

$J_{4422}^{42}$-1-40-1-1-40-1-3211212212-21121-2

$J_{4422}^{43}-10-300-1-2-1111-10-1111202-2110$
$J_{4422}^{44}-2-1000-2-101111101-1010-1-2110$

$J_{4422}^{45}-3-200-5-2002212212-212-1-14-3-10$

$J_{4422}^{46}-2-10-5-20-1-4-221211-321-4133322$

$J_{4422}^{47}-2-10-4-20-1-3-221210-221-3123311$

$J_{4422}^{48} \quad 0-6-20-2-20-1-11-112134122-321-20$

$J_{4422}^{49}-1-3-50-4-10-11-111313-4122331-31$

$J_{4422}^{50}-200-4-20-1-3-22121-1-221-2113311$

$J_{4422}^{51}$ 0-40-1-2-3-10111-2312211-10-3211

$J_{4422}^{52}$-2-4-200-3-4-2222-2313312-11-6321

$J_{4422}^{53}-2-4-200-3-2-2221-3312312-11-4311$

$J_{4422}^{54}-2-100-3-2001211101-112-1-12-200$

$J_{4422}^{55}-3-20-1-5-3001321202-223-2-14-301$

$J_{4422}^{56} \quad 0-2-3-4-20-3-11-431112-2-23213311$

$J_{4422}^{57}-2-40-2-2-300-121122-3413-2-42120$

$J_{4422}^{58}-2-10-2-2-10-241-311-111-31-221121$

$J_{4422}^{59}-1-2-200-2-3-2111-1202212-21-3221$

$J_{4422}^{60} \quad 0-2-1-10-2-1-1-311110111101111-1$

$J_{4422}^{61}$ 0-4-1-10-4-1-1-4211212212-11121-2

$J_{4422}^{62}-1-4-10-1-4-10-3211212212-11121-3$

$J_{4422}^{63} \quad 00-2-2-10-1-31-201-201211-122111$

$J_{4422}^{64}-2-400-1-4-1-2121-1323212-31-4211$

$J_{4422}^{65}-2-4000-4-3-2121-14232-12-31-5231$

$J_{4422}^{66}-10-3000-1-21-211-10-111212-2-111$

$J_{4422}^{67}-2-200-1-1-40121-121211-22-1-3120$

$J_{4422}^{68}-2-4000-4-2-2121-1323202-31-4221$

$J_{4422}^{69} \quad 0-2-1-2-20-101-1101011111-221-11$

$J_{4422}^{70}-20-4000-2-32-322-10-221322-3-121$

$J_{4422}^{71}-500-100-1-2222200-110-211-2111$

$J_{4422}^{72}-20-3000-2-32-221-10-221222-3-121$

$J_{4422}^{73}-3-5-100-5-2-3231-2324313-42-5321$

$J_{4422}^{74}-1-200-1-10-311111011-11-121-1-11$

$J_{4422}^{75}-2-20-100-3-11111112-10-221-2111$

$J_{4422}^{76} \quad 0-1-4-2-3-20022-1-211-2113213-211$

$J_{4422}^{77}-40-2-10-4-102221021-213-21-3211$

$J_{4422}^{78}-3-30-10-4-1-2132-2212203-31-3221$

$J_{4422}^{79}-20-6000-2-32-422-10-221433-3-221$

$J_{4422}^{80}-2-3-200-2-401211312-21-231-423-1$

$J_{4422}^{81}-10-4000-1-21-21100-111222-2-111$

$J_{4422}^{82}-2-2-200-3-1-1121-1211112-11-4211$

$J_{4422}^{83} \quad 00-1-30-2-3-2-2211001-11-3222311$

$J_{4422}^{84} \quad 0-1-6-1-6-30-133-1-211-3224334-411$

$J_{4422}^{85}-4-30-1-4-30-1113214-333-3-11231-2 \quad J_{442}^{128}$

$J_{4422}^{86}$-3-20-1-3-20-1012213-322-3-11221-1
$J_{4422}^{87} \quad 00-1-30-3-3-1-2211011-21-2212311$

$J_{4422}^{88} 00-1-2-1-2-30-1110111-21-2211211$

$J_{4422}^{89} \quad 00-2-40-3-4-2-3212-112-22-3323411$

$J_{4422}^{90}-10-1-3-10-1-32-311-3-21211-121221$

$J_{4422}^{91}-5-20-1-5-20-1223323-423-4-11321-3$

$J_{4422}^{92}-5-20-1-5-20-1123323-423-4-11321-2$

$J_{4422}^{93}-20-2-40-2-30-2212-122-31-2312311$

$J_{4422}^{94}-20-1-3-2-1-20-2211111-21-2212211$

$J_{4422}^{95}-30-2-6-2-1-40-3321112-31-4423422$

$J_{4422}^{96}-20-1-5-2-1-40-3221112-31-3313322$

$J_{4422}^{97}-6-30-1-6-30-1124324-434-4-11331-2$

$J_{4422}^{98}-20-2-50-2-50-3222-123-31-3413321$

$J_{4422}^{99}-20-3-4-200-3-21121-1-332-2322311$

$J_{4422}^{100} \quad 00-2-50-4-6-2-3212-123-42-3423522$

$J_{4422}^{101} \quad 0-2-4-3-5-20032-1-222-2212314-421$

$J_{4422}^{102}-3-100-3-1003112111-211-2-12-2-1-1$

$J_{4422}^{103} \quad 0-3-4-4-5-20042-1-322-2312314-422$

$J_{4422}^{104} 00-3-60-4-7-3-4213-224-43-4534622$

$J_{4422}^{105}-3-30-2-3-30-2412-2112322-42-232-1$

$J_{4422}^{106}$ 00-1-40-2-4-1-2111-112-21-2213321

$J_{4422}^{107} 00-1-40-2-4-2-3112-112-21-2213322$

$J_{4422}^{108}-200-4-200-422-322-3-11-3-1-132133$

$J_{4422}^{109}-8-20-2-8-20-234534-62252-1-432-44$

$J_{4422}^{110}-10-2-3-10-2-3-21121-1-321-3322221$

$J_{4422}^{111} \quad 0-1-3-1-3-10-121-1-111-2112212-211$

$J_{4422}^{112}-2-300-3-10-2211-2122211-212-201$

$J_{4422}^{113}-5-2-10-5-2-10123322-323-301321-3$

$J_{4422}^{114}-60-3-3-1-4-104222121-332-21-5413$

$J_{4422}^{115}-50-2-1-2-4-303233222-422-31-3311$

$J_{4422}^{116}-40-1-1-1-3-202222121-311-21-2211$

$J_{4422}^{117}-5-1-2-10-5-102322131-313-31-3311$

$J_{4422}^{118}-3-1-1-10-3001211021-212-20-2201$

$J_{4422}^{119}-1-4-1-2-6-200321-2122232-314-411$

$J_{4422}^{122}-2-50-2-6-20-1322-3123332-314-411$

$J_{4422}^{121}-20-4-20-30012-1102-1-22220-3311$

$J_{4422}^{122}-30-2-1-2-2-302122212-321-21-2211$

$J_{4422}^{123}-10-6-5-1-4-1012-21121-34222-5433$

$J_{4422}^{124}-200-5-30-2-2-32121-1-112-3214411$

$J_{4422}^{125}-20-2-3-20-2-34-412-4-21311-122321$

$J_{4422}^{126}-1-10-4-20-3-1-1111112-31-3212222$

$J_{4422}^{127} \quad 00-30-30-2-4-3-1121-1-1233222-310$

$00-50-5-2-1-321-1-211-3234432-311$
$00-2-50-2-4-1-3121012-32-2213322$ the algorithm ПB from a highly degenerate facet, since in this case the number of vertices of $P^{\prime}$ which are fed in the cdd program can easily become larger than the number the program can handle. In our specific case we used Brunner and Gisin's $I_{4422}^{19}$ Bell inequality as an input facet to the algorithm and the 129 tight Bell inequalities were obtained using both methods ПA and ПB However, we mention that we did not perform an exhaustive search, since it would have been time consuming. We suspect that the list of 26 inequalities of Ref. [12] plus our 129 introduced ones do not form the complete set of all 4422-type of tight Bell inequalities. This is implied by the fact, that our set of 129 inequalities covered only 23 from the list of 26 inequalities of Brunner and Gisin. The coefficients defining the 129 inequalities are shown in Table【 


\section{LOWER BOUNDS}

The quantum value of a Bell expression is the expectation value of the operator

$$
\begin{aligned}
& \sum_{i=1}^{m_{A}} b_{A_{i}}\left(A_{i} \otimes I_{B}\right)+\sum_{j=1}^{m_{B}} b_{B_{j}}\left(I_{B} \otimes B_{j}\right) \\
& +\sum_{i=1}^{m_{A}} \sum_{j=1}^{m_{B}} b_{A_{i} B_{j}}\left(A_{i} \otimes B_{j}\right) .
\end{aligned}
$$

According to Eqs. (11) and (2) this is the value one gets for the left hand side of Eq. (2) with measurements on a quantum system. This can be larger then the maximum classical value $b_{0}$, the quantum violation is the difference of the quantum value and $b_{0}$. For the maximum quantum violation it is enough to consider pure states.

We have given lower limits for the maximum violation of Bell inequalities by explicitly constructing measurement operators in Alice's and Bob's component Hilbert spaces and the appropriate state using numerical optimization. We have made calculations with six-dimensional real and complex spaces and eight-dimensional real spaces, taking three- and fourdimensional projection operators as measurement operators, respectively. The results of such a calculation may correspond to solutions in component spaces of lower dimensionality. The six-dimensional case incorporates the five-dimensional case, in which each measurement operator can be a two- or a three-dimensional projector, the four-dimensional case with one-, twoand three-dimensional projectors, and the three- and two-dimensional cases with projectors of any dimensionality, including degenerate operators, i.e., zero and unity. The eight-dimensional calculation covers the seven-dimensional case with four- and three-dimensional projectors, the six-dimensional one with four-, threeand two-dimensional projectors, the five-dimensional one with four-, three-, two- and one-dimensional projectors, and the four-, three- and two-dimensional ones with any projectors.

The Schmidt decomposition of the maximally violating state vector tells which subspaces of the component spaces it occupies. The dimensionality of those subspaces is the Schmidt number, the number of nonzero Schmidt coefficients. If this is less than the number of dimensions of the component spaces, and if each measurement operator projects the corresponding subspace onto itself, then the orthogonal subspace may be dropped altogether, reducing the dimensionality of the solution. The latter condition is fulfilled if the matrix of each measurement operator is block-diagonal in the Schmidt basis, such that all matrix elements connecting the subspace the state vector occupies with the orthogonal subspace are zero. Although this was not always true for the results we got from the optimization procedure, it turned out that we could always get rid of the unwanted nonzero matrix elements without changing the optimum value achieved by penalizing them in a subsequent optimization. In these cases there were equally good solutions with different choices of the measurement operators concerned. Thus a Schmidt number smaller than the dimensionality of the component Hilbert space we considered always led to a solution in a smaller space.

The number of dimensions of a measurement operator in the reduced space is given by the dimensionality of the overlap of the subspace it projects to with the subspace the state vector occupies. We can easily get it as the trace of the corresponding block of the matrix of the operator. The dimensionality of the overlap of a five-dimensional and a three-dimensional subspace of a six-dimensional space is either two or three. This is the reason our sixdimensional calculation with three-dimensional projectors may lead to a five-dimensional solution with both two- and three-dimensional measurement operators. To try all these possibilities for a Bell inequality with five measurement settings per party with confining ourselves to five-dimensional component spaces would mean $2^{10}$ separate optimizations. To check all potential fourdimensional solutions a single six-dimensional calculation covers would require $3^{10}$ complete calculations. Although the number of parameters to be optimized is larger, it is still better to work in higher-dimensional spaces.

In our previous work [18] we studied many of the Bell inequalities we consider here, but then we confined ourselves to four-dimensional component spaces with twodimensional projectors. We solved the problem by maximizing the maximum eigenvalue of the $16 \times 16$ matrix of the operator given by Eq. (4) with a simplex uphill method. A difficulty with this approach is that it almost always finds a solution with qubits, even if a better solution exists with higher dimensional component spaces. For some inequalities it finds the true maximum only once in several thousand attempts. With four-dimensional spaces the number of calculations necessary to get reliable results were well affordable. However, in six- or eight-dimensional component spaces it is even more difficult to find the interesting solution, there are a lot more parameters to be optimized, and the size of the matrices to be diagonalized many times in each optimization run is also much larger. Therefore, we have changed our strategy and we have used an iterative approach. The procedure ensures that the Schmidt bases of the state vector agree with the bases used in the component spaces, up to possible phase factors.

When we try to find the best $m$-dimensional solution with the $n$-dimensional ( $n=6$ or 8 ) program, we first take the $m$-dimensional maximally entangled state $1 / \sqrt{m} \sum_{i=1, m}|i\rangle|i\rangle$. We apply the simplex uphill method 32] to find the best measurement operators to maximize the expectation value of the operator (44), that is the quantum value of the inequality with the state chosen. Then we replace the vector with the one of the form $\sum_{i=1, n} c_{i}|i\rangle|i\rangle$ that gives the largest violation with the measurement operators we have got. This vector can directly be determined by finding the eigenvector belong- 
ing to the largest eigenvalue of a matrix. We repeat these steps until we reach convergence. As the vector always has the special form in our basis, the matrices whose expectation value has to be calculated and the ones to be diagonalized are of the size of $n \times n$. We only have to deal with small submatrices of the corresponding matrices of size $n^{2} \times n^{2}$ in the full Hilbert space. For the simplex uphill method we use the implementation of Ref. [33]. The procedure does not ensure to find the true optimum, so we have to repeat it many times. To save computation time we made just a few iterations with many different initial values, and chose only a few of them that arrived at the best values for the full calculation. In another implementation we stopped the iterations as soon as any of the Schmidt coefficients (which are just $\left|c_{i}\right|$ ) fell below a threshold, and restarted the procedure with new initial values.

To apply optimization we have to parametrize the operators. An (n/2)-dimensional projector in an $n$ dimensional space requires $(n / 2)^{2}$ real parameters to characterize in the real case, and twice as many in the complex case. In choosing the parameters we used a similar approach than in Ref. [18]. In Ref. 34] it has been shown that any $(n / 2)$-dimensional projector in an $n$-dimensional space can be transformed into a simple form with two unitary transformations, one acting only on the first $n / 2$, while the other on the other $n / 2$ basis vectors. This simple form is characterized by $n / 2$ angles. Then any such projector can be produced by acting on such a simple matrix with two appropriate unitary - or in the real case orthogonal - transformations. A general three-dimensional orthogonal transformation can be described with 3 Euler angles, while the four-dimensional one requires 6 Euler angles (see for example Ref. 35]). This way we get the correct number of parameters for the projectors in the real case both in the six- $(3+2 \cdot 3=9)$ and in the eight-dimensional $(4+2 \cdot 6=16)$ spaces. In a six-dimensional space each three-dimensional unitary transformation requires further 4 phase factors to be characterized, but one overall factor is irrelevant. The 9 extra parameters we get this way give just the necessary number of parameters for the projectors in the complex case.

\section{UPPER BOUNDS}

To determine upper limits for the maximum quantum violation of the Bell inequalities we follow the procedure given by Navascués et al. [19, 20]. The method, in its simplest form, specified to the case of a two-party twooutcome Bell inequality, is as follows.

Let us introduce the operators $a_{i}=\left(2 A_{i}-I_{A}\right) \otimes I_{B}$ and $b_{j}=I_{A} \otimes\left(2 B_{j}-I_{B}\right)$, where $I_{A}$ and $I_{B}$ are the unity operators in Alice's and Bob's component spaces, respectively. The $a_{i}$ and $b_{j}$ operators have eigenvalues +1 and -1 for the two outcomes of the measurement instead of 1 and 0 . The square of each of them is unity, and ev- ery $a_{i}$ commutes with every $b_{j}$. Let us choose a set of vectors containing $|\psi\rangle, a_{1}|\psi\rangle, a_{2}|\psi\rangle, \ldots, a_{m_{A}}|\psi\rangle, b_{1}|\psi\rangle$, $b_{2}|\psi\rangle, \ldots, b_{m_{B}}|\psi\rangle$, and any number of vectors of the form $a_{i} a_{i^{\prime}} a_{i^{\prime \prime}} \ldots b_{j} b_{j^{\prime}} b_{j^{\prime \prime}}|\psi\rangle$, i.e., $|\psi\rangle$ multiplied by the product of any numbers of $a$ and $b$ operators. Let us consider the matrix whose entries are the real parts of the scalar products of these vectors. A matrix derived this way is positive semidefinite. Each entry of the matrix is the real part of the expectation value of an operator. Taking into account that each $a_{i}$ and $b_{j}$ squares to unity, and that $a_{i}$ commutes with $b_{j}$, one can see that in some places the operators may be equal, or hermitian conjugates of each other. In those places the entries of the matrix will be equal, independently of the actual choice of $|\psi\rangle$ and the measurement operators. It is easy to see that the quantum value for a Bell inequality can be written as a linear combination of certain entries of this matrix. There are limits on the value this linear combination may take, as the matrix has to be positive semidefinite, while satisfying constraints requiring the equality of some of its elements. The quantum value of the Bell inequality can not be larger than the maximum value allowed by the conditions above. The standard optimization method to determine this maximum is semidefinite programming [36]. There are several codes available to solve the problem, in most of our calculations we used CSDP [37.

The upper limit for the maximum violation of the inequality we get this way depends on how we choose the set of vectors we generate the matrix from. Refs. [19, 20] introduce a systematic hierarchy of choices, where the higher levels give ever stricter limits until the exact maximum violation is achieved. The first level includes only the vectors $|\psi\rangle, a_{i}|\psi\rangle\left(i=1, \ldots, m_{A}\right)$ and $b_{j}|\psi\rangle$ $\left(j=1, \ldots, m_{B}\right)$. The second level also includes vectors $a_{i} a_{i^{\prime}}|\psi\rangle, b_{j} b_{j^{\prime}}|\psi\rangle$ and $a_{i} b_{j}|\psi\rangle\left(i, i^{\prime}=1, \ldots, m_{A}\right.$, $\left.j, j^{\prime}=1, \ldots, m_{B}\right)$, that is it includes all vectors we can make by acting on $|\psi\rangle$ with up to two measurement operators. We get level three by going up to three operators, and so on. The exact value for the maximum violation may be reached at a finite level, for correlation type Bell inequalities this already happens at the first level [38]. It has been shown that the series of upper limits through the hierarchy converges to the exact value [20, 41]. In practice, it is also worth considering intermediate levels. The computation requirement is often much less than that is for the next full level, and if it gives the exact value, we need not go any further. For example, for many of the Bell inequalities we considered we got the upper limit equal to the lower one by supplementing level two by just the vectors generated with two $a$ and one $b$ (noted as $\left.a a^{\prime} b\right)$, or one $a$ and two $b$ operators $\left(a b b^{\prime}\right)$.

\section{RESULTS FOR THE MAXIMUM VIOLATION}

Tables $\Pi$ and $\amalg$ show our results for the maximum violation of the Bell inequalities we could achieve by explic- 
TABLE II: Maximum violation of Bell inequalities. The identitity $\mathcal{H}$ of the component Hilbert spaces, the dimensionalities $D_{A_{i}}$ and $D_{B_{j}}$ of the measurement operators of the participants, and the level $\mathrm{L}$ when the upper limit reaches the value of violation is also shown. Notation $1 \mathrm{a}, 2 \mathrm{a}$ and $2 \mathrm{~b}$ mean level 1 plus $a b$, level 2 plus $a a^{\prime} b$ (or $a b b^{\prime}$ ) and level 2 plus $a a^{\prime} b+a b b^{\prime}$, respectively. An alternative notation for $I_{3322}, I_{3422}^{2}$ and $I_{4422}^{1}$ is $A_{3}, A_{4}$ and $A_{7}$, respectively.

\begin{tabular}{|c|c|c|c|c|c|c|c|c|c|c|c|c|c|c|c|c|c|}
\hline & & & $D_{A_{i}}$ & $B_{j}$ & $\mathrm{~L}$ & & & $\mathcal{H}$ & $D_{A_{i}}$ & $D_{B_{j}}$ & $\mathrm{~L}$ & & & $\mathcal{H}$ & $D_{A_{i}}$ & $D_{B_{j}}$ & $\mathrm{~L}$ \\
\hline$I_{3322}$ & 2500000 & $\mathbb{R}^{2}$ & 111 & 111 & no & & 0.4571068 & $\mathbb{C}^{2}$ & 0111 & 11111 & $2 \mathrm{a}$ & & & $\mathbb{R}^{2}$ & 11111 & (1111 & $2 \mathrm{a}$ \\
\hline$I_{3422}^{1}$ & & $\mathbb{R}^{2}$ & 011 & 1111 & $1 \mathrm{a}$ & I. & & $\mathbb{R}^{2}$ & 1111 & 01111 & 2 & 4 & & $\mathbb{R}^{2}$ & & & $2 \mathrm{a}$ \\
\hline$I_{3422}^{2}$ & 2990381 & $\mathbb{R}^{2}$ & 111 & & $2 a$ & & & $\mathbb{R}^{2}$ & 1111 & & $1 \mathrm{a}$ & $A_{55}$ & & $\mathbb{R}^{2}$ & & & $1 \mathrm{a}$ \\
\hline$I_{3422}^{3}$ & 4364917 & $\mathbb{R}^{2}$ & 111 & 1111 & $1 \mathrm{a}$ & & & $\mathbb{C}^{2}$ & 1111 & & $2 \mathrm{a}$ & & & $\mathbb{R}^{2}$ & & & $2 \mathrm{a}$ \\
\hline$A_{5}$ & & $\mathbb{R}^{2}$ & 1111 & 111 & $2 a$ & & & $\mathbb{C}^{2}$ & & & $2 \mathrm{a}$ & & & $\mathbb{R}^{2}$ & & & $2 \mathrm{a}$ \\
\hline$A_{6}$ & & $\mathbb{R}^{4}$ & 1222 & & 3 & & & $\mathbb{R}^{5}$ & 2242 & & no & & & $\mathbb{R}^{2}$ & & & $2 \mathrm{a}$ \\
\hline$A S_{1}$ & & $\mathbb{R}^{2}$ & 1111 & & 1 & & & $\mathbb{R}^{2}$ & 1111 & & $2 \mathrm{a}$ & & & $\mathbb{R}^{2}$ & & & $2 \mathrm{a}$ \\
\hline$A S_{2}$ & & $\mathbb{R}^{2}$ & 1111 & & 1 & & & $\mathbb{C}^{2}$ & 11111 & & 2 & & & $\mathbb{R}^{5}$ & & & $2 b$ \\
\hline$A I I_{1}$ & & $\mathbb{R}^{2}$ & 1111 & & $1 \mathrm{a}$ & & & $\mathbb{R}^{2}$ & 111 & & $1 \mathrm{a}$ & & & $\mathbb{C}^{2}$ & & & $2 \mathrm{a}$ \\
\hline$A I I_{2}$ & & & 1 & & 2 & & & $\mathbb{R}^{2}$ & & & $1 \mathrm{a}$ & & & $\mathbb{R}^{5}$ & & & no \\
\hline$I_{4422}^{1}$ & 3 & $\mathbb{R}^{3}$ & 1111 & & $2 \mathrm{a}$ & & & $\mathbb{C}^{2}$ & & & 2 & & & $\mathbb{R}^{4}$ & & & \\
\hline$I_{4422}^{2}$ & 712 & $\mathbb{R}^{2}$ & & & 2 & & & $\mathbb{R}^{2}$ & & & 2 & & & $\mathbb{R}^{3}$ & & & \\
\hline$I_{4422}^{3}$ & 6 & $\mathbb{R}^{2}$ & 2111 & & $1 \mathrm{a}$ & $A$ & & $\mathbb{R}^{2}$ & & & $1 \mathrm{a}$ & & & $\mathbb{R}^{5}$ & & & no \\
\hline$I_{4422}^{4}$ & 136 & $\mathbb{R}^{2}$ & 1100 & & $2 \mathrm{a}$ & & & $\mathbb{C}^{2}$ & & & 2 & & & $\mathbb{C}^{2}$ & & & \\
\hline$I_{4422}^{5}$ & 436 & $\mathbb{R}^{2}$ & 111 & & $1 \mathrm{a}$ & & & $\mathbb{R}^{2}$ & & & $1 \mathrm{a}$ & & & $\mathbb{R}^{5}$ & & & no \\
\hline$I_{4422}^{6}$ & 7 & $\mathbb{C}^{2}$ & 1111 & & $1 \mathrm{a}$ & & & $\mathbb{R}^{2}$ & & & $1 \mathrm{a}$ & & & $8^{5}$ & & & no \\
\hline$I_{4422}^{7}$ & 3 & $\mathbb{C}^{2}$ & 1111 & & 2 & & & $\mathbb{C}^{2}$ & & & $2 \mathrm{a}$ & & & $\mathbb{R}^{2}$ & & & \\
\hline$I_{4422}^{8}$ & 1 & $\mathbb{R}^{3}$ & 2121 & & $2 \mathrm{~b}$ & & & $\mathbb{C}^{2}$ & & & $2 \mathrm{a}$ & & & $\mathbb{R}^{2}$ & & & 2 \\
\hline$I_{4422}^{9}$ & 2 & $\mathbb{R}^{2}$ & 1111 & & $2 \mathrm{a}$ & & & $\mathbb{R}^{2}$ & & & $2 \mathrm{a}$ & $A$ & & $\mathbb{R}^{2}$ & & & \\
\hline$I_{4422}^{10}$ & 6 & $\mathbb{R}^{2}$ & 1111 & & $2 \mathrm{a}$ & & & $\mathbb{R}^{2}$ & & & $2 \mathrm{a}$ & $A$ & & $\mathbb{R}^{2}$ & & & \\
\hline$I_{4422}^{11}$ & 6383543 & $\mathbb{R}^{2}$ & 1111 & & $1 \mathrm{a}$ & & & $\mathbb{C}^{2}$ & & & $2 \mathrm{a}$ & & & $\mathbb{R}^{2}$ & & & \\
\hline$I_{4422}^{12}$ & 42 & $\mathbb{R}^{2}$ & 1111 & & $2 \mathrm{a}$ & & & $\mathbb{C}^{2}$ & & & $2 \mathrm{a}$ & & & $\mathbb{R}^{2}$ & & & \\
\hline$I_{4422}^{13}$ & 53 & $\mathbb{R}^{2}$ & 1112 & & $2 \mathrm{a}$ & $A_{3}$ & & $\mathbb{C}^{2}$ & 11111 & & $2 \mathrm{a}$ & & & $\mathbb{R}^{2}$ & & & $2 \mathrm{a}$ \\
\hline$I_{4422}^{14}$ & 3 & $\mathbb{R}^{2}$ & 1110 & & $2 \mathrm{a}$ & & & $\mathbb{C}^{2}$ & 11111 & & 2 & & & $\mathbb{R}^{3}$ & & & $2 a$ \\
\hline$I_{4422}^{15}$ & 3 & $\mathbb{R}^{2}$ & 1011 & & $2 \mathrm{a}$ & & & $\mathbb{R}^{2}$ & & & $1 \mathrm{a}$ & & & $\mathbb{R}^{2}$ & & & $2 a$ \\
\hline$I_{4422}^{16}$ & 36 & $\mathbb{R}^{2}$ & 1011 & & $1 \mathrm{a}$ & & & $\mathbb{C}^{2}$ & & & $2 \mathrm{a}$ & $A$ & & $\mathbb{R}^{2}$ & & & 2 \\
\hline$I_{4422}^{17}$ & 85 & $\mathbb{R}^{2}$ & 1111 & 11 & $2 \mathrm{a}$ & & & $\mathbb{R}^{2}$ & & & $1 \mathrm{a}$ & & & $\mathbb{C}^{2}$ & & & $2 \mathrm{a}$ \\
\hline$I_{4422}^{18}$ & 0 & $\mathbb{R}^{3}$ & 1111 & & $2 a$ & & & $\mathbb{R}^{2}$ & 11111 & & $1 \mathrm{a}$ & & & $\mathbb{R}^{4}$ & & & no \\
\hline$I_{4422}^{19}$ & 07 & $\mathbb{R}^{3}$ & 2112 & 2122 & $2 \mathrm{~b}$ & & & $\mathbb{R}^{2}$ & 11211 & & $2 \mathrm{a}$ & & & $\mathbb{C}^{2}$ & & & $2 \mathrm{a}$ \\
\hline$I_{4422}^{20}$ & 794 & $\mathbb{R}^{4}$ & 2223 & 2223 & no & & & $\mathbb{C}^{2}$ & 11111 & & $2 \mathrm{a}$ & $A_{\varnothing}$ & & $\mathbb{R}^{4}$ & & & no \\
\hline$A_{8}$ & 501 & $\mathbb{C}^{2}$ & 1111 & 11111 & $1 \mathrm{a}$ & & & $\mathbb{R}^{5}$ & 23333 & & $2 \mathrm{~b}$ & $A_{\varepsilon}$ & & $\mathbb{C}^{2}$ & & & $2 \mathrm{a}$ \\
\hline$A_{9}$ & 428 & $\mathbb{C}^{2}$ & 1111 & 11111 & $2 \mathrm{a}$ & & & $\mathbb{C}^{2}$ & 11111 & & no & $A_{\varepsilon}$ & & $\mathbb{R}^{4}$ & & & no \\
\hline$A_{10}$ & 0.415 & $\mathbb{R}^{5}$ & 3233 & 23333 & $2 \mathrm{~b}$ & & & $\mathbb{R}^{4}$ & 11222 & & $2 \mathrm{a}$ & $A$ & & $\mathbb{C}^{2}$ & & & $2 \mathrm{a}$ \\
\hline$A_{11}$ & 0.456 & $\mathbb{C}^{2}$ & 1111 & 11111 & $2 \mathrm{a}$ & & & $\mathbb{C}^{2}$ & 11111 & 11111 & $2 \mathrm{a}$ & $A_{86}$ & & $\mathbb{C}^{2}$ & & & $2 \mathrm{a}$ \\
\hline$A_{12}$ & 0.487 & $\mathbb{C}^{2}$ & 1111 & 11111 & 2 & & & $\mathbb{C}^{2}$ & 11111 & 11111 & $2 \mathrm{a}$ & $A_{87}$ & & $\mathbb{R}^{4}$ & & & $2 \mathrm{a}$ \\
\hline$A_{13}$ & & & 1222 & 12222 & $2 \mathrm{a}$ & & & $\mathbb{R}^{2}$ & 11111 & & $2 \mathrm{a}$ & $A_{8}$ & & & & & 2 \\
\hline$A_{14}$ & 0.4758457 & & 3322 & 22232 & no & $A_{52}$ & 0.6218611 & $\mathbb{R}^{2}$ & 11111 & 11111 & $2 \mathrm{a}$ & $A$ & 0.3025898 & $\mathbb{R}^{6}$ & 22343 & 22343 & 110 \\
\hline & & & 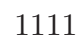 & 11 & & & & & & & & & & & & & \\
\hline
\end{tabular}

itly determining the state vector and the measurement operators. The size of the component Hilbert space and the dimensionalities of the operators are also shown. In our earlier work [18] we have determined the maximum violation for all cases shown in Table II with component Hilbert spaces of up to four dimensions taking twodimensional projectors as measurement operators. For the inequalities we follow the notations we used there, which are the same as the ones in Refs. [11, 12]. By extending our calculations to six-dimensional complex and real, and eight-dimensional real component spaces we could achieve a larger maximum violation for 19 of the 112 inequalities. We found 9 cases where fourdimensional real spaces were necessary for maximum vi- 
TABLE III: Maximum violation of Bell inequalities introduced in the present paper The identitity $\mathcal{H}$ of the component Hilbert spaces, the dimensionalities $D_{A_{i}}$ and $D_{B_{j}}$ of the measurement operators of the participants, and the level L when the upper limit reaches the value of violation is also shown. Notation $1 \mathrm{a}, 2 \mathrm{a}$ and $2 \mathrm{~b}$ mean level 1 plus $a b$, level 2 plus $a a^{\prime} b\left(\right.$ or $\left.a b b^{\prime}\right)$ and level 2 plus $a a^{\prime} b+a b b^{\prime}$, respectively.

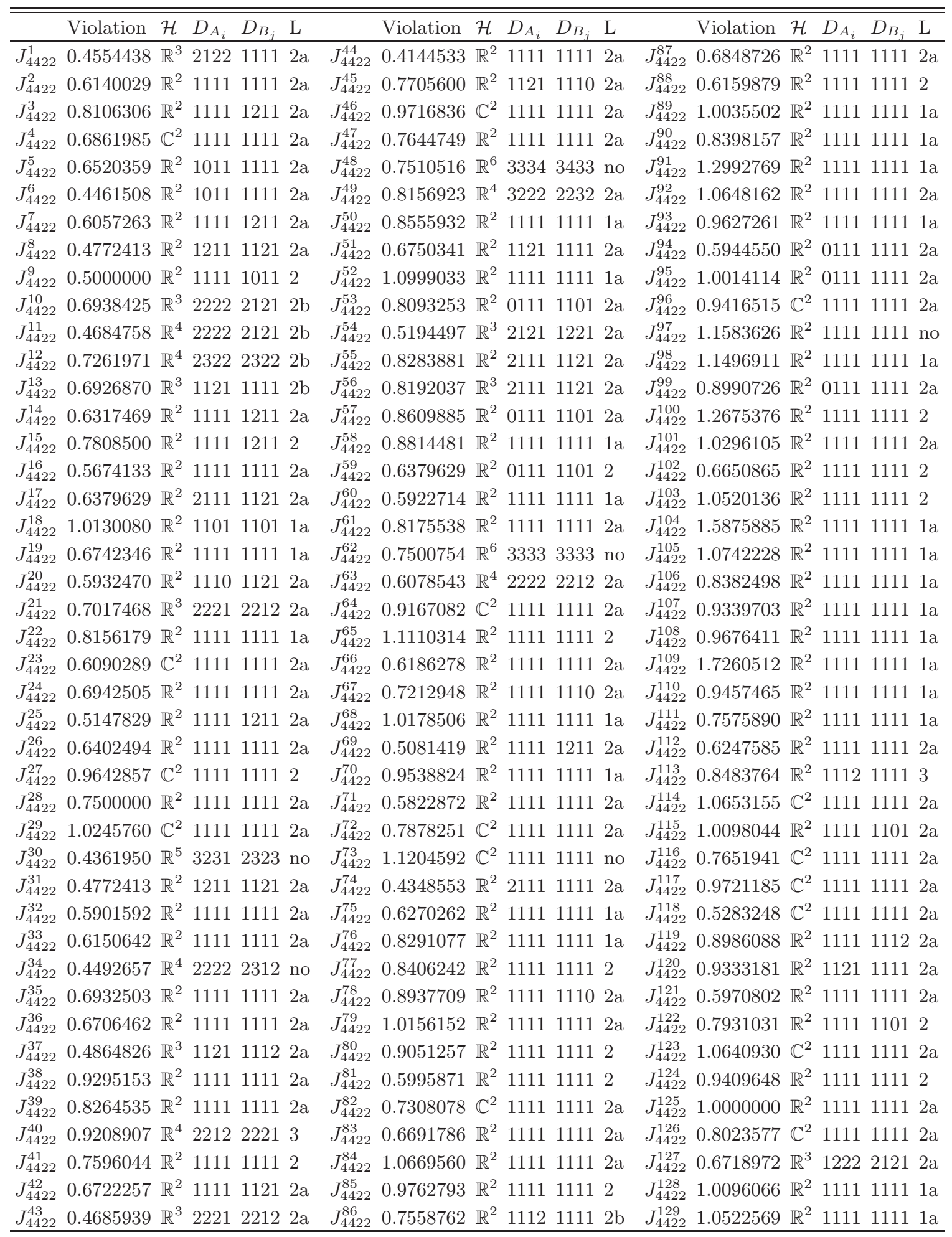


TABLE IV: Difference between the upper limit calculated at different levels and the lower limit derived by explicitly constructing the measurement operators.

\begin{tabular}{|c|c|c|c|c|c|c|c|}
\hline Case & $L 2$ & $L 2+a a^{\prime} b$ & $\begin{array}{c}L 2+85 \% \text { of } \\
a a^{\prime} b+a b b^{\prime}\end{array}$ & $\begin{array}{c}L 2+a a^{\prime} b+ \\
+a b b^{\prime}\end{array}$ & $\begin{array}{c}L 2+a a^{\prime} b+ \\
a b b^{\prime}+a a^{\prime} a^{\prime \prime}\end{array}$ & $L 3$ & $\begin{array}{c}L 3+a a^{\prime} a^{\prime \prime} b+ \\
a a^{\prime} b b^{\prime}+a b b^{\prime} b^{\prime \prime}\end{array}$ \\
\hline$I_{3322}$ & 0.0009397 & 0.0008863 & & 0.0008760 & 0.0008756 & 0.0008756 & 0.0008754 \\
\hline$I_{4422}^{20}$ & 0.0393814 & 0.0111147 & & 0.0040799 & & 0.0000784 & \\
\hline$J_{4422}^{30}$ & 0.0401921 & 0.0109109 & & 0.0042758 & & 0.0010680 & \\
\hline$J_{4422}^{34}$ & 0.0240714 & 0.0064382 & & 0.0023265 & & 0.0001503 & \\
\hline$J_{4422}^{48}$ & 0.0294613 & 0.0014661 & & 0.0008068 & & 0.0006361 & \\
\hline$J_{4422}^{62}$ & 0.0022695 & 0.0000194 & & 0.0000178 & & 0.0000167 & \\
\hline$J_{4422}^{73}$ & 0.0329957 & 0.0030318 & & 0.0023042 & & 0.0020987 & \\
\hline$J_{4422}^{97}$ & 0.0392054 & 0.0018759 & & 0.0005867 & & 0.0001425 & \\
\hline$A_{14}$ & 0.0274655 & 0.0057079 & & 0.0031815 & 0.0025615 & & \\
\hline$A_{21}$ & 0.0048537 & 0.0007595 & & 0.0005165 & 0.0003840 & & \\
\hline$A_{47}$ & 0.0202626 & 0.0050055 & 0.0026688 & & & & \\
\hline$A_{62}$ & 0.0133913 & 0.0031907 & 0.0025556 & & & & \\
\hline$A_{64}$ & 0.0164779 & 0.0032730 & 0.0016572 & & & & \\
\hline$A_{65}$ & 0.0175447 & 0.0014105 & 0.0000806 & & & & \\
\hline$A_{67}$ & 0.0135307 & 0.0019890 & 0.0006118 & & & & \\
\hline$A_{68}$ & 0.0073408 & 0.0039257 & 0.0038884 & & & & \\
\hline$A_{80}$ & 0.0161885 & 0.0026707 & 0.0006052 & & & & \\
\hline$A_{82}$ & 0.0082846 & 0.0065568 & 0.0064928 & & & & \\
\hline$A_{84}$ & 0.0271831 & 0.0040203 & 0.0021524 & & & & \\
\hline$A_{89}$ & 0.0104965 & 0.0043258 & 0.0033485 & & & & \\
\hline
\end{tabular}

olation, with not all projectors two-dimensional. For another 9 inequalities we had to go up to five-dimensional real component spaces. One of them is $A_{10}$, a 4522 inequality, which has less than five measurement settings for one of the parties. For one 5522 we found the maximum violation with six-dimensional component spaces, more than the number of measurement settings for either party. Similarly, for $J_{4422}^{30}$ (see Table III), we needed fivedimensional, and for $J_{4422}^{48}$ and $J_{4422}^{62}$, all 4422 cases, sixdimensional real component spaces. However, for these cases we can not be sure that the states and the operators we constructed does give the true quantum bound. The values for the violation are still somewhat below the corresponding upper limits we have got. Some possibilities, even with lower dimensional component spaces, like fourdimensional complex spaces with allowing degenerate measurement operators are not covered. Moreover, we may have even missed some solutions which are in principle accessible by our programs. The six-dimensional program with complex spaces often struggles to find solutions it finds relatively easily in real spaces. This is not very surprising, as in the complex case there are twice as many parameters to optimize. We note that whenever we needed more than two-dimensional component spaces for maximum violation for the inequalities considered in the present paper, real spaces were always sufficient. This is not true for all Bell inequalities, for example for the 4822 correlation type inequality introduced in Ref. [40] the lowest dimensional component spaces to achieve the maximum violation are four-dimensional complex ones. Some complex solutions may have been missed by our present calculations, but not many of them, as for the great majority of the inequalities the solution we managed to construct are actually as good as they can be, according to the upper limit we found.

For the cases the upper limit agreed with the lower one derived from the explicit construction, we also show in Tables II and III the (usually partial) level when it happened. For those inequalities the maximum violations shown are the exact values. The level we could afford to accomplish with the code and with the computers we used depended on the number of measurement settings. For the 5522 inequalities we could do the calculation with either all $a a^{\prime} b$ or $a b b^{\prime}$ type vectors added to level two, but with not both of them. Therefore, the largest calculations we made included only the $85 \%$ of them, randomly chosen. For the 4522 cases the maximum we could do was level two plus $a a^{\prime} b+a b b^{\prime}+a a^{\prime} a^{\prime \prime}$ (i.e., level 3 without $\left.b b^{\prime} b^{\prime \prime}\right)$. For 4422 we could go up to level 3 , while for 3322 to level three plus $a a^{\prime} b b^{\prime \prime}+a a^{\prime} a^{\prime \prime} b+a b b^{\prime} b^{\prime \prime}$. We had to go to the maximum we could afford only for a small fraction of the inequalities. For over $85 \%$ of the cases level two plus $a b b^{\prime}$ (and for the same cases level two plus $a a^{\prime} b$ ) has already given the exact value.

There were 20 inequalities for which the upper limit remained above the best solution we found (marked by 
no in the Tables) at all levels we could afford. The gap between the upper and lower limits for those inequalities, depending on the level is shown in Table IV] In almost all of these cases extending the calculation for the higher limit further decreases this gap, often quite significantly. For $I_{4422}^{20}, J_{4422}^{30}, J_{4422}^{34}, J_{4422}^{97}, A_{65}, A_{67}$ and $A_{80}$ it seems to be probable that the lower limits we got are exact. When the decrease of the gap is relatively very small, like in the cases of $J_{4422}^{73}, A_{68}$ or $A_{82}$, it is more likely that we still have not got the true solution.

However, we find the case of $I_{3322}$ truly puzzling. This is one of the simplest tight Bell inequalities, with just three measurement settings for each party. In our formulation used in (2) for the Bell inequalities it reads

$$
\begin{aligned}
& -2 p_{A_{1}}-p_{A_{2}}-p_{B_{1}}+p_{A_{1} B_{1}}+p_{A_{1} B_{2}}+p_{A_{1} B_{3}} \\
& +p_{A_{2} B_{1}}+p_{A_{2} B_{2}}-p_{A_{2} B_{3}}+p_{A_{3} B_{1}}-p_{A_{3} B_{2}} \leq 0 .
\end{aligned}
$$

We could calculate the upper limit at a level significantly exceeding level 3 (up to level 3 the upper limit has also been calculated by Refs. [20, 41]). The gap between the lower limit of 0.25 and the upper limit is at least 4 orders of magnitude larger than numerical uncertainty, while it gets just marginally smaller by the extension of the calculation for the upper limit. It does not behave as if it would decrease much further. The lower limit has already been achieved with real two-dimensional component spaces. All our attempts to find a larger violation with higher dimensional spaces failed, although the number of parameters is much smaller than for the other inequalities we considered, and we have also made a lot more attempts than for any other case. It does not seem very likely for us that with spaces and operators our calculations cover such a solution exists. At the same time, it would also be very surprising, if one needed even higher dimensional Hilbert spaces to violate maximally this very simple inequality.

Our present results confirm what we have found in Ref. [18], namely in most cases the state giving the maximum violation is not the maximally entangled one (indication for this fact was also given independently by Ref. [12]). The results here are stronger, because the comparison with the upper bound proves that for most inequalities the states and operators we have got are actually the ones giving the absolute quantum bound. When the component Hilbert spaces required were larger than two-dimensional, the state we found was never the maximally entangled one. For bipartite inequalities with two measurement settings, but more than two outcomes 39], a similar result was found by Ref. [19].

\section{DETECTION EFFICIENCIES}

A loophole-free experiment in order to test the nonlocal nature of quantum mechanics is still missing [7]. In particular, none of the experiments performed to date could close simultaneously the locality loophole (the measure- ment results at Alice's and Bob's side should be spacelike separated) and the detection loophole. In order to avoid this latter loophole the particles must be detected with a high enough probability, otherwise a locally causal model can reproduce the measured correlations. Another motivation beside the fundamental ones comes from the security issue of some quantum communication protocols which is based on the loophole-free violation of Bell inequalities [42].

There are different proposals to close the detection loophole. More than two outcomes [43, 44], more than two settings 12, 24, 43 45], and partially entangled states [24, 46, 47] has been considered as well. The case of asymmetric Bell experiments, where the two particles are detected with different probabilities (in systems such as entangled atom-photon pairs) has also been addressed 12, 24, 47]. Another promising approach is the application of homodyne detectors in Bell tests [48 50].

Here we calculate both the symmetric (Alice and Bob have the same efficiency $\eta_{A}=\eta_{B} \equiv \eta$ ) and asymmetric (Alice's detector is perfect) threshold detection efficiencies of our set of Bell inequalities for a pair of maximally entangled qubits allowing degenerate measurements as well. The general approach, consisting of two different detection efficiencies $\left(\eta_{A}\right.$ and $\left.\eta_{B}\right)$ was treated in Ref. [24]. According to their Eq. (4) the quantum value of a Bell inequality with detection efficiencies $\eta_{A}$ and $\eta_{B}$ is given by

$$
\begin{aligned}
I_{\eta_{A}, \eta_{B}} & =\eta_{A} \eta_{B} Q+\eta_{A}\left(1-\eta_{B}\right) M_{A} \\
& +\left(1-\eta_{A}\right) \eta_{B} M_{B}+\left(1-\eta_{A}\right)\left(1-\eta_{B}\right) X
\end{aligned}
$$

where $Q$ is the quantum value associated to the Bell inequality with perfect detectors, $M_{A, B}$ and $X$ are the values when one or both detectors do not fire. Next we limit ourselves to maximally entangled states and in the case of no detection one of the two outcomes $\{1,0\}$ are taken as an output. If we consider degenerate measurements as well, the no-detection outcomes must be set to the same value as the output of the corresponding degenerate measurement. On the other hand, by fixing these values the original Bell inequality $I$ reduces to another one $I^{\prime}$ with a smaller number of settings. The local bound $L$ for this inequality can be smaller or equal to the local bound corresponding to the Bell inequality $I$. At the same time it can be observed that the values $M_{A, B}$ and $X$ remain the same for $I^{\prime}$. Thus, if for a given Bell inequality $I$ and efficiencies $\eta_{A}, \eta_{B}, I_{\eta_{A}, \eta_{B}}>L$ holds, it certainly holds true for the reduced Bell inequality $I^{\prime}$ as well. This implies that the threshold efficiencies for $I^{\prime}$ can only be lowered with respect to the original one $I$ (in our actual calculations we found that $L$ never decreased, hence leaving unchanged the detection efficiencies). Conversely, this result means that if one supplements a Bell inequality with coefficients pertaining to degenerate measurements (i.e., deterministic ones, which need not be performed at all), it cannot lower the detection efficiency. Note, that this result, which is intuitively clear, also holds for the case of partially entangled states. We made an optimiza- 
tion over all possible measurement strategies (i.e., for any combination of degenerate and non-degenerate measurements) and over all possible no-detection outcomes, both in the symmetric and asymmetric situations. The following results have been obtained:

From the literature [24] for the maximally entangled, asymmetric case the best known detection efficiency is $\eta_{B}=2 / 3$, corresponding to $I_{3322}$. We found that inequality $I_{3422}^{2}$ and $A I I_{2}$ yield the same value with nondegenerate measurements. With involving degenerate measurements as well, this threshold value was obtained for a further 97 inequalities. Out of them 69 can be traced back to $I_{3322}$, while 12 is reducible to non-tight 3322 inequalities, nonequivalent with $I_{3322}$. However, $\eta_{B}=2 / 3$ is not the best one can achieve, for three five-setting Bell inequalities from the list of Avis et al. [11], namely, $A_{34}, A_{44}$, and $A_{50}$ do better. The respective values of $\eta_{B}=0.6607,0.6520,0.6587$ are reached with purely non-degenerate measurements. There exists a local model [51], which reproduces the correlations arising from non-degenerate projective measurements which can be expressed using real (complex) numbers on the maximally entangled state under the assumption that Alice has perfect detector and Bob has detection efficiency $\eta_{B}=2 / 3\left(\eta_{B}=1 / 2\right)$. Thus the three lower than $2 / 3$ values given above need to belong to projective measurements with settings requiring complex numbers to be described. This fact, as we have checked, is true.

For the maximally entangled symmetric case, the threshold efficiency for the CHSH inequality is known to be 0.8284 , which result was slightly improved recently by Brunner and Gisin [12]. They showed that the $A_{5}$ inequality from the Avis et al. list allows the slightly smaller threshold of 0.8214. By computing the best threshold values for the set of 241 Bell inequalities we did not find any better case. Allowing degenerate measurements we could get the same value for 19 five-setting Bell inequalities, but they could all be traced back to $A_{5}$.

\section{SUMMARY}

Let us summarize the main results achieved in this work.

We have presented two heuristic methods to get tight two-party two-outcome Bell inequalities. Using these methods we have extended the list of known 4422 type inequalities by 129 members. The list is very probably still not full.

For 241 inequalities we used numerical optimization to determine measurement operators and states giving their maximum quantum violation achievable with sixdimensional complex component Hilbert spaces, allowing three-dimensional projectors as measurement operators, and with eight-dimensional real component Hilbert spaces, allowing four-dimensional projectors as measure- ment operators. An $n$-dimensional calculation with $(n / 2)$-dimensional projectors covers all $m$-dimensional $(m \leq n)$ cases with projectors having any dimensionality between $\max (0, m-n / 2)$ and $\min (m, n / 2)$. The 241 examples we have considered include all tight bipartite Bell inequalities with up to five two-outcome measurement settings known to us (excluding $\mathrm{CHSH}$, about which we could tell nothing new). These results represent lower limits for the quantum bounds for the maximum violation. At the same time, the method proposed in Refs. [19, 20] makes it possible to determine a series of upper bounds by carrying out the calculation through a hierarchy of levels. The series converges to the exact value, often reaching it at a finite level. For the majority of cases the upper bound has become equal to the lower one at a level which is quite easy to perform for inequalities with no more than five settings per party. There remained only 20 cases for which we still can not tell for sure the maximum value of their quantum violation. The lower limit is probably the exact value for quite a few of them, but unfortunately we could not do the calculation for the upper limit at a high enough level to verify it. It is surprising that for the smallest case we considered, with only three measurement settings per party, there is still a significant gap between the upper and lower bounds, while the former seems to come only marginally lower by increasing the level, and for the latter we were unable to find any better solution than one can get with real qubits, despite the high dimensionality of the component spaces we considered and the many attempts we have done. We have only found a few inequalities whose maximum violation was achieved with the maximally entangled state, in all those cases a pair of qubits were sufficient.

We have also calculated the minimum detection efficiency to verify quantum violation for each inequality with a maximally entangled pair of particles, both for symmetric and asymmetric arrangements. By allowing degenerate measurements (ones with a certain outcome), one could lower these threshold efficiencies for most inequalities, but this only means that the (not necessarily tight) inequality with less measurement settings one can get by skipping the degenerate ones is at least as good at verifying quantum violation. As far as the actual numbers are concerned, for the symmetric case none of the inequalities does better than the best one already known, while for the asymmetric case we have found three inequalities with five measurement settings per party, for which slightly less efficient detectors are sufficient than for the best one known so far.

\section{Acknowledgments}

Many thanks to Stefano Pironio for pointers to the literature. T.V. has been supported by a János Bolyai Grant of the Hungarian Academy of Sciences. 
[1] J.S. Bell, Physics 1, 195 (1964).

[2] J.F. Clauser, M.A. Horne, A. Shimony, and R.A. Holt, Phys. Rev. Lett. 23, 880 (1969).

[3] A. Aspect, P. Grangier, G. Roger, Phys. Rev. Lett. 47, 460 (1981); G. Weihs et al., Phys. Rev. Lett 81, 5039 (1998); W. Tittel, J. Brendel, H. Zbinden, and N. Gisin, 81, 3563 (1998); M.A. Rowe et al., Nature (London) 409, 791 (2001).

[4] L.A. Khalfin, B.S. Tsirelson, Found. Phys. 22, 879 (1992).

[5] B.S. Tsirelson, Hadronic Journal Supplement 8, 329 (1993).

[6] B.S. Tsirelson, problem 33 (2006), presented in the web page http://www.imaph.tubs.de/qi/problems.

[7] N. Gisin, arXiv:quant-ph/0702021 (2007).

[8] B.S. Tsirelson, Lett. Math. Phys., 4, 93 (1980).

[9] L.A. Khalfin, B.S. Tsirelson, Quantum and quasiclassical analogs of Bell inequalities. Symposium on the Foundations of Modern Physics, edited by Lahti et al. (World Scientific, Singapore, 1985), pp 441-460.

[10] S. Popescu and D. Rohrlich, Found. Phys., 24, 379 (1994).

[11] T. Ito, H. Imai, and D. Avis, List of Bell inequalities for at most 5 measurements per party via triangular elimination (2006), available in the web page www-imai.is.s.u-tokyo.ac.jp/ ${ }^{\sim}$ tsuyoshi/bell/bell5.html D. Avis, H. Imai, T. Ito, and Y. Sasaki, J. Phys. A: Math. Gen. 38, 10971 (2005).

[12] N. Brunner and N. Gisin, Phys. Lett. A 372, 3162 (2008).

[13] M. Froissart, Nuov. Cim. B, 64, 241, (1981).

[14] C. Sliwa, Phys. Lett A 317, 165 (2003).

[15] D. Collins and N. Gisin, J. Phys. A: Math. Gen. 37, 1775 (2004).

[16] D. Collins (unpublished).

[17] T. Ito, H. Imai, and D. Avis, Phys. Rev A 73, 042109 (2006).

[18] K.F. Pál and T. Vértesi, Phys. Rev. A 77, 042105 (2008).

[19] M. Navascués, S. Pironio, and A. Acín, Phys. Rev. Lett. 98, 010401 (2007).

[20] M. Navascués, S. Pironio, and A. Acín, New J. Phys. 10, 073013 (2008).

[21] N. Brunner, S. Pironio, A. Acín, N. Gisin, A.A. Méthot, and V. Scarani, Phys. Rev. Lett. 100, 210503 (2008).

[22] D. Pérez-García, M.M. Wolf, C. Palazuelos, I. Villanueva, and M. Junge, Comm. Math. Phys. 279, 455 (2008).

[23] S. Wehner, M. Christandl, and A.C. Doherty, Phys. Rev. A 78, 062112 (2008).

[24] N. Brunner, N. Gisin, V. Scarani, C. Simon, Phys. Rev. Lett. 98, 220403 (2007).

[25] R. Cleve, P. Høyer, B. Toner, and J. Watrous, Proceedings of the 19th IEEE Annual Conference on Computational Complexity (CCC 2004), p. 236-239; arXiv:quant-ph/0404076 (2004).

[26] I. Pitowsky, Mathematical Programming 50, 395 (1991).

[27] The program cdd by Komei Fukuda is a $\mathrm{C}++$ implementation of the Double Description Method of Motzkin et al. 28], which solves both the convex hull and vertex enumeration problems. The cdd package is available from the web site of Fukuda: www.cs.mcgill.ca/ fukuda/soft/cdd_home/cdd.html.

[28] T.S. Motzkin, H. Raiffa, G.L. Thompson, and R.M. Thrall, The double description method. Contributions to theory of games, Vol. 2. (Princeton University Press, New Jersey, Princeton, 1953).

[29] I. Pitowsky, Quantum Probability, Quantum Logic, Lecture Notes in Physics 321, (Heidelberg, and Springer, 1989).

[30] R. Seidel, Constructing Higher-Dimensional Convex Hulls at Logarithmic Cost per Face. STOC 86, 404 (1986).

[31] Ll. Masanes, Quantum Information and Computation 3, 345 (2002).

[32] J.A. Nelder and R. Mead, Comput. J., 7, 308 (1965).

[33] W.H. Press, S.A. Teukolsky, W.T. Vetterling, and B.P. Flannery, Numerical Recipes: The Art of Scientific Computing, 3rd ed. (New York: Cambridge University Press, 2007).

[34] B. Toner and F. Verstraete, arXiv:quant-ph/0611001 v1 (2006).

[35] N.Ja. Vilenkin, Special Functions and the Theory of Group Representations, Trans. Math. Monographs, Vol. 22 (Am. Math. Soc., Providence, R.I., 1968).

[36] L. Vandenberghe and S. Boyd, SIAM Rev. 38, 49 (1996).

[37] B. Borchers, CSDP, A C Library for Semidefinite Programming. Optimization Methods and Software 11, 613, (1999); https://projects.coin-or.org/Csdp/.

[38] S. Wehner, Phys. Rev. A, 73, 022110 (2006).

[39] D. Collins, N. Gisin, N. Linden, S. Massar, and S. Popescu, Phys. Rev. Lett. 88, 040404 (2002).

[40] T. Vértesi and K.F. Pál, Phys. Rev. A 77, 042106 (2008).

[41] A.C. Doherty, Y.-C. Liang, B. Toner, and S. Wehner, Proceedings of the 23rd Annual IEEE Conference on Computational Complexity (CCC 2008), p. 199-210; arXiv:0803.4373 (2008).

[42] J. Barrett, L. Hardy, and A. Kent, Phys. Rev. Lett. 95, 010503 (2005); A. Acín, N. Gisin, and Ll. Masanes, Phys. Rev. Lett. 97, 120405 (2006); A. Acín, N. Brunner, N. Gisin, S. Massar, S. Pironio, and V. Scarani, Phys. Rev. Lett. 98230501 (2007).

[43] S. Massar, S. Pironio, J. Roland, and B. Gisin, Phys. Rev. A 66, 052112 (2002).

[44] J.H. Bigelow, arXiv:0805.0387 (2008).

[45] H. Buhrman, P. Høyer, S. Massar, and H. Röhrig, Phys. Rev. Lett. 91, 047903 (2003).

[46] P.H. Eberhard, Phys. Rev. A 47, R747 (1993).

[47] A. Cabello and J.-A. Larsson, Phys. Rev. Lett. 98, 220402 (2007).

[48] R. García-Patrón, J. Fiurášek, N.J. Cerf, J. Wenger, R. Tualle-Brouri, and Ph. Grangier, Phys. Rev. Lett. 93, 130409 (2004).

[49] M. Paternostro, H. Jeong, T.C. Ralph, Phys. Rev. A 79, 012101 (2009).

[50] A. Acín, N.J. Cerf, A. Ferraro, J. Nisen, Phys. Rev. A 79, 012112 (2009).

[51] N. Gisin and B. Gisin, Phys. Lett. A 260, 323 (1999).

[52] www .atomki.hu/atomki/TheorPhys/Bell_violation/. 